\title{
América Latina: saberes ancestrales en la producción del cacao
}

\section{RESUMEN}

Msc. Gilmer José Maco Luján

gilmerml@gmail.com

ORCID: 0000-0002-9421-459X

Universidad César Vallejo

Msc. Luz del Carmen Correa Ayambo

lucorrea779@gmail.com

ORCID: 0000-0002-1721-7295

Universidad César Vallejo

Dr. José Manuel Delgado Bardales

jmdelgadob@ucvvirtual.edu

ORCID: 0000-0001-6574-2759

Scopus Author ID: 24070333700

Código Renacyt: P0050554

Universidad César Vallejo

La investigación tuvo como objetivo, identificar las barreras de la transferencia del conocimiento en el cultivo de Cacao nativo en el Trapecio Amazónico desde la valoración de la diversidad del saber milenario para su protección. Investigación básica, descriptiva, con revisión sistemática. Se tomaron 14 artículos científicos y 12 entrevistas para determinar las barreras en la trasferencia de los conocimientos ancestrales a los comuneros de la localidad de Cushillococha en Mariscal Ramón Castilla. Resultados: Se encontraron que las primeras barreras, están orientadas a la migración de los hijos hacia las urbes, lo cual conlleva a la ausencia del actor que recepciona el conocimiento, por otro lado, la escuela no coadyuva en esta transición y el rol de los líderes comunales se va perdiendo entre las nuevas adopciones de costumbres en este proceso de transculturalidad. Conclusiones: Se requiere del apoyo de la entidad educativa y otros servicios para fortalecer los conocimientos del cultivo nativo del cacao en el Trapecio Amazónico, para lo cual urgen políticas más focalizadas a este desarrollo.

Palabras clave: identificar; saberes ancestrales; pueblos nativos; conocimientos milenarios. 


\title{
Latin America: ancestral knowledge in cocoa production
}

\begin{abstract}
The objective of the research was to identify the barriers to the transfer of knowledge in the cultivation of native Cacao in the Amazon Trapezoid from the valuation of the diversity of millenary knowledge for its protection. Basic, descriptive research, with systematic review. 14 scientific articles and 12 interviews were taken to determine the barriers in the transfer of ancestral knowledge to the community members of the town of Cushillococha in Mariscal Ramón Castilla. Results: It was found that the first barriers are oriented to the migration of children to the cities, which leads to the absence of the actor who receives the knowledge, on the other hand, the school did not contribute to this transition and the role of the children. Community leaders are getting lost among the new adoptions of customs in this process of transculturality. Conclusions: The support of the educational entity and other services is required to strengthen the knowledge of the native cultivation of cacao in the Amazon Trapezoid, for which more focused policies are urgently needed for this development.
\end{abstract}

Keywords: identify; ancestral knowledge; native peoples; ancient knowledge.

Artículo recibido: 05 octubre. 2021 Aceptado para publicación: 02 noviembre 2021 Correspondencia: gilmerml@gmail.com Conflictos de Interés: Ninguna que declarar 


\section{INTRUDUCCIÓN}

Considerando los conocimientos de los sembríos, gracias al vínculo entre la naturaleza y el hombre que es indesligable; esta cosmogonía andina, considera a la fiesta del calendario andino, el calendario lunar, en las actividades agrarias de los pueblos indígenas. Las nuevas generaciones tienen que aprender los saberes ancestrales, orientado a la tierra, siembra y cosecha; así mismo agradecen ofreciendo agradecimiento a la Pachamama (Madre Tierra en Español), como lo afirma (Global Network Content Services LLC, DBA Noticias Financieras LLC, 2017)"; a partir que se puede argumentar que los hábitos y costumbres en las comunidades indígenas se transmiten de padres a hijos, para aprovechar los recursos naturales que ofrece la naturaleza a sus hijos, en este escenario de supervivencia el ser humano tiene que ingeniárselas para aprovechar los recursos que le ofrece la naturaleza; gracia a ello se puede observar los aporte de la agricultura a la alimentación, como la medicina se ha enriquecido y usa el conocimiento para crear medicinas como la penicilina, y todos argumentos le debemos y es herencia invalorable de los saberes ancestrales; y como agradecimiento a lo que recibe el hombre de la naturaleza le rinde tributo de diferentes formas a través de la Madre Tierra, costumbre que está enraizada en las sociedad andina y amazónica.

Los saberes ancestrales en salud divulgan la utilización de las plantas medicinales así como las curaciones posibles de realizar con las técnicas y el saber local heredado de padres a hijos en las comunidades nativas de la amazonia, lo que se plasma en la realidad es que los pueblos indígenas de las regiones amazónicas, de Ayacucho, Cuzco, Loreto y otros han registrado 6585 registro de conocimientos colectivos de usos y propiedad de recursos biológicos ante INDECOPÍ, lo cual se construyó con el saber ancestral que está constituido por los saberes que han adquirido los pueblos y comunidades indígenas y que para su supervivencia se transmiten de padres a hijos, en una cadena interminable y eterna, desde el primer hombre que habito nuestro territorio hasta nuestros hermanos aguarunas, shipibos en la región Amazónica. "Los resultados que arrojan el vínculo entre la educación en sus idioma natal y la forma como transmiten sus saberes en el proceso de vivencia local y a partir de allí consolidar su cultura sostenida por sus costumbres que lo ayuda a su supervivencia en un mundo globalizado, postula (Corporación Universitaria Minuto de Dios - UNIMINUTO, 
2017)", donde tienen que defender sus derechos y territorio que con el desarrollo de las ciudades ve peligrar su morada y su territorio indígena, en este devenir los organismos internacionales mediante convenios a lo cual está suscrito el Perú, el cual protege a los pueblos indígenas y sus saberes ancestrales. La cultura inca con su saber ancestral heredado a nuestra cultura la papa, el camote, maní, maíz; particularmente la papa ha salvado de la hambruna a la humanidad, en periodos críticos de la sociedad.

Los saberes ancestrales no son invulnerable están sujetos al flujo y reflujo del individuo, que muchas veces ve peligrar su calidad de vida, genera más complejidad en su cosmovisión, aun el individuo en su comunidad está sujeto a la influencia de sus semejantes que en su accionar, crea nuevas expectativas de mejoras o estabilidad para su vivencia en su ambiente natural, Rengifo-Salgado, et al. (2017), señala que, las comunidades o pueblos tienen saberes que se transmiten de padres a hijos, y cada líder de un pueblo o comunidad adquiere los saberes de su experiencia diaria en contacto con la naturaleza y la influencia de su entorno, la que ofrece soluciones inmediatas o problemas complejos que ameritan dedicación; los saberes ancestrales se enriquecen y fortalecen con la técnica y conocimiento científico a lo cual puede acudir el poblador indígena; el poblador está en contacto con la sociedad y allí recibe influencia que ayuda a comprender y desarrollar mejor sus habilidades y competencias adquiridas en su proceso de interrelación con el mundo; así mismo es necesario precisar que los medio para comunicarse con sus semejantes es el lenguaje, herramienta que los ayuda a consolidar su cultura y costumbres en un escenario marcado por proteger a la naturaleza y a las comunidades indígenas, que demanda respeto de su cultura y territorio.

La educación que se brinda a las comunidades indígenas debe ser en su idioma natal, para que puedan comprender y facilitar la adquisición y transmisión de conocimientos y técnicas, que necesitan para lograr una vida satisfactoria. El saber ancestral es patrimonio de los pobladores que habitan un territorio, conformado por usos y costumbres que en su proceso histórico en su aprehensión con la naturaleza adquiere nuevas habilidades, como a pescar determinados peces en una zona que alberga algunas plantas. (ContentEngine LLC, a Florida limited liability company, 2019), desde sus inicios la supervivencia humana está marcada por luchas y dominio de la naturaleza, en este contacto donde el poblador en su afán de agricultor, pescador, adquiere ese valioso saber ancestral que en contacto con el exterior se trastoca en una educación científica 
intercultural, amerita a su esfuerzo por conquistar el mundo, coadyuvan a preservar si bagaje cultural, lingüística, historia e identidad; así mismo el uso del saber ancestral en la agricultura es lo que le ha ayudado más a consolidar su posición de conquistador y aliado de la naturaleza; en las comunidades indígenas en cacao orgánico por su fuerte demanda en los mercados internacionales es el producto para exportar, basado el enfoque de desarrollo, propuso Amartya Sen, considera tres enfoques: el primero el desarrollo personal, el segundo la integración en la sociedad y el tercero referido a la sostenibilidad. Esta posición considera a los productores del cacao como: individuos receptores y generadores de ingresos y saberes ancestrales basados en el cultivo del cacao; como comunidad integrados a la cultura con sus costumbres; sociedad de desarrollo sostenible, conservadores de la biodiversidad ancestral, que han heredado de sus antepasados.

Afirma la (UNESCO 2020) "Los conocimientos ancestrales se refieren al saber generacional y habilidades practicadas en su devenir histórico en comunión con su entorno" El saber indígena debe ser conservado y difundido en su realidad práctica, que conlleve beneficios a la sociedad utilitaria. Dueñas y Aristizábal, (2017, pág. 40), se ha considerado incorporar nuevos saberes y prácticas culturales, sobre la realidad social y la diversidad cultural”. Es importantes incorporar el saber ancestral en los estudios sobe la realidad local y su influencia cultural. Agrega Meléndez y Mendoza (2018) "El predomino del 'procedimiento en la alfarería ancestral de los pedidos de alfarería del pueblo de Huancas, 2017" Tipo de estudio: descriptiva. Población: tres poblaciones. Muestra: 119 personas. Cuestionario: marcas de artesano con la que fabrican alfarería en Huancas, el $75 \%$ es alfarería, $20 \%$ textilería, el $5 \%$ madera y el $0 \%$ otros. Procedimientos que utilizan para la hacer la cerámica, el $80 \%$ es los procedimientos ancestrales, el $15 \%$ procedimiento de cerámica al frio, el $5 \%$ procedimiento de cerámica vidriada, el $0 \%$ otros.

Conclusión: la tecnología alfarera ancestral utilizada por los pueblos si influye en diminutas equidades, ya que sus productos son adquiridos en baja proporción, por carecer de una difusión.

Argumenta Ramos (2019), "En su investigación Utilización de los conocimientos milenarios en cuidados de sistemas de salud de los comuneros de Acoria. Método de investigación: etnográfico. Tipo de experimentación: básica, descriptiva. Diseño: no 
experimental. Población: 502 personas. Muestra: 37 personas. Técnica de investigación: observación, entrevista. Enfermedades comunes más atendidas sobrenaturales $37.04 \%$, gracias a quien conoce los conocimientos ancestrales a mamá un 50\%, identifica las cualidades curativas de las plantas medicinales un 100\% algunos. Conclusión: la utilización de los conocimientos milenarios, para el cuidado de la salud es parte del sincretismo andino-occidental”. Nugkuag (2017), “Las comunidades nativas amazónicas y el reto de ejercer actividades económicas bajo el enfoque del desarrollo de identidad. Tipo y diseño de experimentación: cualitativa. Población y muestra: 60 personas. Ilicitudes que padecen los poblados para lograr un desarrollo económico con identidad: no encontrar mercado para vender sus productos. Conclusión: se determinó una fuerte base moral que comparte bajo un sistema de cosmo visión intelectual común en las comunidades".

Astete, (2017) "La ruta ancestral inka camino al Antisuyo, posee un gran potencial turístico para diversificar la oferta turística de la ciudad Imperial. Su infraestructura en su periodo histórico gozada de modernidad que hasta la fecha causa admiración su nivel de ingeniería". Ramos Huamán, Luz Maritza,(2018). "Para la sociedad indígena los conocimientos ancestrales acerca del cuidado del bienestar, es parte de la cosmovisión andina heredada de sus ancestros; basada en sus conocimientos y prácticas cotidianas, cuyo fruto la madre tierra les provee, como una bendición divina". (Salgado, Sandra, Mlaverri, \& Vargas, 2016)" La teoría del saber ancestral se origina en la práctica, en sus chacras, donde se obtiene datos de sus plantas". Es necesario investigar sobre los beneficios de las plantas, para de allí elaborar medicinas que nos ayudaran la restablece la salud de los individuos que deambulan por el medio ambiente".

Ruiz Tacuri Ruth Elizabeth, (2016) “La identidad de la cultura indígena se cohesiona en sus moradores, que enfrentan los inevitables e influyentes cambios; que los pobladores deben asimilar como herramienta para dominar la naturaleza, que a diario les presenta retos a ser dominados". (Suarez, 2019) "La adopción de los saberes ancestrales al sistema educativo moderno, debe ser evaluada bajo el criterio pragmático y científico en concordancia con la ética; para incorporarlos a la saber educativo y académico universitario". (Melendez \& Mendoza, 2018) "La venta de sus artesanías ha caído debido, a la carencia de promoción de sus artesanías ofrecido a los turistas, que visitan su pueblo". Se necesita capacitar y promocionar los productos de la zona citada y crear 
un paquete turístico que impulse a la zona como una región turística. (Bonilla, 2018) "Auspiciar lugares de asistencia de la población nativa, para recapacitar en la identidad socio cultural en su territorio; la mejora que debe hacerse y el saber que debe difundirse, como pueblo inmerso en la sociedad moderna". Estimar la cultura local, con sus costumbres, códigos.

(Niño, 2018) "Los indígenas perdieron el control de sus tierras, fueron obligados a trabajar como peones, e integraron el nuevo campesinado desposeído".

Sin embargo, el gobierno colombiano tomo medias serias para el bienestar de la población nativa. (Uribe, 2019), "El saber ancestral hay que enfocarlo desde una posición no eurocéntrica, que trascienda el cientificismo para redimir el saber indígena, como variables válidas para incrementar el conocimiento". El saber ancestral está latiendo en las comunidades a espera de ser conocido para generar conocimiento. (Rengifo, Ríos, Fachin, \& Vargas, 2017) "Se recuperó conocimiento y datos de la comunidad, y ejecuto talleres dirigido a los pobladores". El conocimiento debe difundirse en pro del desarrollo, para beneficio de los pueblos indignas y de su entorno; consolidando la información con datos cuantitativos, lo que permite analizar y llegar a conclusiones válidas para tomar decisiones, en el contexto territorial. (DW, 2018) "El saber indígena es clave para diseñar estrategias de adaptación y el diseño de planes para minimizar riesgos y alertar contingencias". En base al conocimiento se diseñan estrategias que consolidaran al saber ancestral en la sociedad moderna. (Pavón, 2018) "La concepción occidental se trastoca en realidad en las naciones indígenas a través del colonialismo"

El colonialismo en el Perú, diezmo la población indígena, sumiéndola en una dependencia de sobrevivencia y explotación, dándole trabajo para que subsista en la agricultura y minas, almacenaba y transportaba a España la riqueza extraída de tierras peruanas. Analiza el papel de los saberes indígenas de la Gente de Centro -grupo indígena multiétnico de la Amazonia colombiana- en el Plan de salvaguarda étnico uitoto capítulo Leticia (PSE). Este es uno de los 34 planes que el Estado colombiano, obligado por la Corte Constitucional, ha formulado e implementado para proteger a los pueblos indígenas amenazados por el desplazamiento forzado. Con base en la investigación anticolonial y de la descolonización, y en la perspectiva subalterna de los derechos humanos, se analiza el PSE como un diálogo intercultural inequitativo en el 
que los pueblos indígenas son obligados a expresarse según los discursos de derechos del Estado y de la Corte.(Hernández Delgado, 2015)

Con este objetivo, introduzco inicialmente el contexto de la Gente de Centro y del Plan de salvaguarda étnica uitoto capítulo Leticia (PSE). Posteriormente, presento el concepto de saberes indígenas basado en perspectivas anticoloniales y de la descolonización. El siguiente relato describe al "niño lombriz" -quien personifica el saber de los uitoto. Este niño [el saber] va a crecer para todos. Él va a repartir, va a hacer todo, quiere decir como un niño, va a tener poder para todo y este niño tiene de todo. Este va a dar, va a repartir consejo, va a repartir historia, canciones, va a repartir más hombre sabedor. Es este niño. Él sabe de todo, de todo. La abuela dice que es lombriz. Su nombre es lombriz, pero no es lombriz. Ese es su hijo, la lombriz ciega que dice, ya quedó historia de la lombriz ciega. El hijo de lombriz es el que va a dar la sabiduría [...]La lombriz realmente estaba engendrando un hijo que era el de la abundancia. Conclusión ¿Cuál es el valor de los saberes indígenas en los procesos de reconocimiento de derechos de pueblos históricamente marginados? El caso de la Gente de Centro en el PSE uitoto capítulo Leticia refleja que los saberes indígenas -como el concepto de abundancia y su conexión con la palabra como elemento creador- han favorecido una visión del PSE como un proceso comunitario sagrado comprometido con el bien común de los participantes en el Plan.(Hernández Delgado, 2015)

(Pérez, Perseita 2016)" En la creencia ancestral el conocerse a si mismo es un proceso de actos experimentales, donde se encuentra las raíces a través de su cosmovisión". La cosmovisión indígena explica y fundamenta sus creencias, ritos, costumbres y saber, extraído de su quehacer diario. (Suárez, 2019) "Los modos de producción han sido heredado de sus ancestros por el homo sapiens, se van construyendo en su interrelación con la naturaleza". El hombre antiguo y moderno han obtenido conocimiento de sus experiencias diarias, en su interrelación con su medio ambiente; que le presenta desafíos, como la deforestación, contaminación de los ríos, explotación indiscriminada de los bosques; quedando el indígena como un espectador de lo que sucede en su territorio.

(Martinez \& López, 2018) "La creación de reservas naturales es una buena decisión para proteger la flora y fauna, y es obligación humana salvaguardar estos recursos que viene a constituir patrimonio nacional y cultural", Las reservas naturales constituyen 
espacios seguros para que la flora y fauna se desarrolle en su verdadera extensión, teniendo como amigo al mortal y enemigo al individuo; amigo a quien busca coger sus fruto racionalmente y enemigo al individuo que explota irracionalmente sus árboles y peces, extinguiendo determinadas especies de su territorio y ríos. Las reservas naturales son creadas por ley, y se da esa enorme tarea a moral, que protege las reservas naturales según su conveniencia o según la envergadura de sus valores; o como extrae el oro de la reserva natural de Marinosqui en Puerto Maldonado - Perú, lo que es un secreto a voces. Por ello se formuló como problema: ¿Cuáles son las características de los Saberes ancestral en la producción del cacao en América Latina? Y como objetivo: Caracterizar los Saberes ancestral en la producción del cacao en América Latina.

\section{ESTRATEGIAS METODOLÓGICAS O MATERIALES Y MÉTODOS}

El tipo de investigación fue básica descriptiva con revisión sistemática de alcance en América Latina desarrollada según la obtención de datos requerido por la publicación. Se usó el método Cochrane para revisiones sistemáticas, uso de la fuente de Investigación secundaria a fin de poder, seleccionar la información pertinente de mayor calidad; para tal efecto se consideró artículos científicos de impacto mundial y regional indexados en data base: Scopus, Web of Sciencie, Redalyc, Latindex, Scielo, Ebsco, y DOAJ, siguiendo los criterios de ubicación en los aspectos: a) plan de búsqueda direccionado en usar los recursos informáticos, que nos ofrece internet en el data base, catálogos de publicaciones indexadas, motor de búsqueda; todas estas herramientas coadyuvarán a consolidar la heurística y profundizar la epistemología en el campo del control interno; b) Inclusión, artículos científicos relacionada a la temática, texto completo, antigüedad 2017 al 2021, idioma español e inglés. c) Exclusión Artículos científicos mayores a cinco años de antigüedad, artículos no relacionados con el tema a investigar y artículos con resumen. El proceso de selección de los estudios fue realizado por dos revisores independientes, y cualquier divergencia fue resuelta por un tercer revisor. Los estudios se seleccionaron por dos pasos. El primer paso es revisar los títulos y resúmenes de las referencias encontradas con nuestra estrategia de búsqueda; se seleccionaron los estudios potencialmente elegibles. El segundo paso consistió en revisar el texto completo de los estudios preseleccionados para confirmar su elegibilidad. 


\section{RESULTADOS Y DISCUSIÓN}

\subsection{Resultados}

Se viene conservando los conocimientos ancestrales del cultivo nativo del cacao, desde la transferencia de conocimiento de los líderes comunales en el trapecio amazónico.

Rengifo-Salgado, et al. (2017) señala que: "Los saberes ancestrales en salud divulgan la utilización de las plantas medicinales, así como las curaciones posibles de realizar con las técnicas y el saber local heredado de padres a hijos en las comunidades nativas de la amazonia.” (p.19), Los pueblos indígenas de las regiones amazónicas, de Ayacucho, Cuzco, Loreto y otros han registrado 6585 tipos de conocimientos colectivos de usos y propiedad de recursos biológicos inscritos ante INDECOPI, lo cual se construyó con el saber ancestral que está constituido por los saberes que han adquirido los pueblos y comunidades indígenas y que para su supervivencia se transmiten de padres a hijos, en una cadena interminable y eterna, desde el primer hombre que habito nuestro territorio hasta nuestros hermanos aguarunas, shipibos en la región Amazónica. Esta transferencia de conocimiento actualmente se viene debilitando por la injerencia de las costumbres occidentales que se constituyen en una barrera para las comunidades nativas que históricamente se dedicaban al cultivo del Cacao nativo, tal como comenta un líder comunal: "es importante recuperar el conociendo ancestral de los pueblos amazónicos para conocer el manejo del cultivo cacao, porque en la frontera no es muy conocido, pero ahora viendo es muy importante” (Líder, Edwing Dávila Dagua, 2021).

"Los saberes ancestrales no son invulnerable están sujetos al flujo y reflujo del individuo, que muchas veces ve peligrar su calidad de vida, genera más complejidad en su cosmovisión, aun el individuo en su comunidad está sujeto a la influencia de sus semejantes que en su accionar, crea nuevas expectativas de mejoras o estabilidad para su vivencia en su ambiente natural, argumenta (ContentEngine LLC, a Florida limited liability company, 2020)". Del mismo modo la influencia de extranjeros por el trapecio Amazónico, afecta las costumbres Tikunas, tal como lo menciona un líder comunal: "La primera dificultad que existe como etnia Tikuna, son la existencia de personas que viene de otros lugares con la finalidad de querer cambiar nuestra cultura y trabajos, por personas foráneas” (Líder, Antolin Turima Santos, 2021).

"El saber ancestral es patrimonio de los pobladores que habitan un territorio, conformado por usos y costumbres que en su proceso histórico en su aprehensión con la 
naturaleza adquiere nuevas habilidades, como a pescar determinados peces en una zona que alberga algunas plantas". (ContentEngine LLC, a Florida limited liability company, 2019)". Afirma la (UNESCO 2020) "Los conocimientos ancestrales se refieren al saber generacional y habilidades practicadas en su devenir histórico en comunión con su entorno" El saber indígena debe ser conservado y difundido en su realidad práctica, que conlleve beneficios a la sociedad utilitaria.

Nugkuag (2017), "Las comunidades nativas amazónicas y el reto de ejercer actividades económicas bajo el enfoque del desarrollo de identidad. Tipo y diseño de experimentación: cualitativa. Población y muestra: 60 personas. Ilicitudes que padecen los poblados para lograr un desarrollo económico con identidad: no encontrar mercado para vender sus productos. Conclusión: se determinó una fuerte base moral que comparte bajo un sistema de cosmo visión intelectual común en las comunidades". "Se hicieron plantaciones con el ministerio de agricultura, ya tenemos cacao, están produciendo, como no hay compradores, entonces no le daban importancia, los que siembran caco le dan facilidades de comercialización, aquí hay el interés por continuar produciendo” (Líder, Marcelo Farian Gaitan, 2021).

Plantea (UNESCO 2020) “Comprender que la población nativa posee formas propias de entender la naturaleza, modos de conservación y formas de manejar los recursos naturales". El poblador que mejor comprende y domina su territorio es el que vive en él. Refiere (UNICEF 2018) "El mayor índice de crecimiento se da en el 2020 con un 61.1 y un índice de desarrollo humano del 0.49 en la comunidad Ngäbe en Panamá" Los datos estadísticos ayudan a plasmar políticas y planes para proteger el saber ancestral de la comunidad indígena.

Menciona (ONU 2020) "Los pueblos indígenas, tienen derechos a decidir su futuro, a su plena autonomía, y a su gobierno en su localidad y a la conservación de su cultura”. Los convenios internacionales protegen a los pueblos indígenas y su cultura, a conservar su territorio". Afirma (CEPAL 2020) "Los pueblos indígenas luchan por reivindicar sus derechos existen dos antecedentes: Convenio de Pueblos nativos y Tribales 1989 y Aclaración de las USA basado en los derechos de los Comunidades Nativas 2007”. Ha sido imperativo proteger a los pueblos vulnerables; solo por presión y luchas se ha logrado. Considera (MIDIS 2019) "Los pueblos indígenas, tienen prioridad en el proceso de inclusión social que se desarrolla, en el Perú; especialmente en las familias 
creadoras de emprendimiento que generen ingresos sostenibles y por ende contribuyan al progreso de su calidad de bienestar". Los convenios internacionales obligan a los países a invertir en el progreso de su calidad de bienestar de la población indígena.

Destaca (Ministerio de Cultura 2020) "En base a la información proporcionada por ENAHO 2019, el 17\% jefe de hogar señalaron no contar con el servicio de agua". Cerrar las brechas es prioridad para generar desarrollo. Describe (Congreso de la República 2020) "Los pueblos nativos tienen derecho a desarrollarse en la esfera, cultural social, económica y política bajo el amparo de la certificación mundial de los derechos civiles".

Sus derechos conquistados en la comunidad internacional es paradigma de constancia y tenacidad.

Define (MIDIS 2018) "Metodología que procesa la pesquisa de las magnitudes físicas y anímicas; lo que permite establecer estratos acordes a su vulnerabilidad y riesgo" Es importante tener un data, de la cual obtengamos información para construir programas encaminados a ayudar los pueblos indígenas más vulnerables a los cambios que genera la globalización. Postula (Ministerio de Agricultura) "Se capacito a las comunidades indígenas con el propósito de brindarles y plantear herramientas para la reactivación de la actividad forestal en concordancia con las medidas sanitarias". Quienes mejor pueden proteger los bosques es la comunidad que la habita, haciendo uso de su conocimiento práctico.

\subsection{Discusión}

La estrategia de búsqueda en este caso dio como resultado 11, 690, en administrado por EBSCOhost, en SCOPUS se obtuvo 49 resultados, academic onefile, se encontró 152, registros publicados, en proQuest, se obtuvo 2,538 resultados. De las cuales 110 fueron revisada, siendo que se logró identificar las más resaltantes según el logro de los resultados de la investigación, y se eliminaron el resto de referencias que no cumplían los criterios de inclusión tras revisar el título y el resumen. Las 110 referencias seleccionadas fueron leídas para confirmar la elegibilidad, logrando considerar 14 artículos para el análisis.

El desarrollo de las competencias de la investigación en los comuneros evaluados sobre si tienen conocimiento del proyecto en su zona de intervención del proyecto y los beneficio que este traería a los miembros de la cooperativa copailet, se dijo que si tiene 
el pleno conocimiento de la existencia del proyecto, que se cuenta con 481 hectáreas sembradas. También tienen presente que el estado peruano llega y tiene presencia por parte del PEBDICP del MDAGRI, por medio del proyecto Binacional del cacao Nativo Fino de Aroma en el Trapecio Amazónicos.

Los trabajos a realizar son Carencias y falta de destrezas de los beneficiarios finales; Pertinencia en relación a carencias y falencias de los grupos beneficiarios finales; Procesos participativos para garantizar la presencia y beneficiarios final. La metodología de intervención será "aprender haciendo", que considera los conocimientos tradicionales, lecciones aprendidas e innovación tecnológica para la construcción y/o mejora de los conocimientos y su aplicación en la mejora del proceso productivo y organizativo. Teniendo como escenario de aprendizaje los talleres de capacitación y las escuelas de campo, con un enfoque innovador para la educación de adultos, de acuerdo a la experiencia sistematizada por la FAO. En las escuelas de campo participarán especialistas en el manejo agronómico y fitosanitario del cacao, así como las diferentes cooperativas y/o federaciones de productores de cacao y demás instituciones del gremio de ambos países.

\section{CONCLUSIONES}

Es importante recuperar el conociendo ancestral de los pueblos amazónicos para conocer el manejo activo del cultivo cacao, para garantizar el mayor beneficio del producto como alimento y producto comercial.

La dificultad que existe en las diferentes etnias es la existencia de personas que vienen de otros lugares con la finalidad de querer cambiar la cultura y trabajos agrícolas, y no valorar sus costumbres.

En varios territorios bajo la responsabilidad del Estado se ha promovido plantaciones de cacao, existe una adecuada producción sin embargo no se cuenta con compradores porque aún las organizaciones no generan asocio y cadenas de valor que aseguren exportar sus productos, es importante que los agricultores tengan acceso a las empresas de comercialización para que incremente interés por continuar produciendo. 


\section{REFERENCIAS BIBLIOGRÁFICAS.}

Abarca, R. (2017). Obtenido de Repositorio Universidad Nacinal San Anatonio Abad del

Cuzco:

http://repositorio.unsaac.edu.pe/bitstream/handle/UNSAAC/2815/253T2017118 3.pdf? sequence $=1 \&$ isAllowed $=\mathrm{y}$

Astete, I. (2017). Obtenido de Epositorio UNASAAC: http://repositorio.unsaac.edu.pe/bitstream/handle/UNSAAC/2815/253T2017118 3.pdf? sequence $=1 \&$ isAllowed $=y$

Baes de datos de pueblos indigenas u originarios. (7 de 12 de 2020). Obtenido de Base de datos de pueblos indígenas u originarios: https://bdpi.cultura.gob.pe/glosario BBC. (4 de Julio de 2020). Obtenido de BBC.COM: https://www.bbc.com/mundo/noticias-52915114

Bonilla, L. (2018). Obtenido de Sentido y prácticade saberes ancestrales: https://ridum.umanizales.edu.co/xmlui/bitstream/handle/20.500.12746/3362/Tesi s_Liliana_Maria_Bonilla.pdf?sequence $=3 \&$ isAllowed $=y$

CEPAL. (2020). Obtenido de CEPAL: https://www.cepal.org/sites/default/files/news/files/folleto_amazonia_posible_y _sostenible.pdf

Coronel, R. (3 de Setiembre de 2020). Obtenido de Dase UNACH: http://dspace.unach.edu.ec/bitstream/51000/7065/1/UNACH-EC-FCEHT-TGP.EDUC-2020-000054.pdf

ContentEngine LLC, a Florida limited liability company. (2019). Obtenido de Proquest: https://www.proquest.com/wire-feeds/los-taitas-y-mamas-salasakas-compartensaberes/docview/2312467027/se-2?accountid=37408

ContentEngine LLC, a Florida limited liability company. (2020). Obtenido de Proquest: https://www.proquest.com/wire-feeds/cómo-ha-afectado-la-actual-pandemia-decovid-19/docview/2437766148/se-2?accountid=37408

Corporación Universitaria Minuto de Dios - UNIMINUTO. (2017). Obtenido de Proquest: https://www.proquest.com/scholarly-journals/relaciones-y-tensionesentre-la-etnoeducación-los/docview/2114614938/se-2?account(2018). Obtenido de

Dispace.unitru: http://www.dspace.unitru.edu.pe/bitstream/handle/UNITRU/11888/Mera\%20An 
drade\%20Rafael\%20Isa\%c3\%adas.pdf?sequence $=1 \&$ isAllowed=y

Abarca, R. (2017). Obtenido de Repositorio Universidad Nacinal San Anatonio Abad del

Cuzco:

http://repositorio.unsaac.edu.pe/bitstream/handle/UNSAAC/2815/253T2017118 3.pdf? sequence $=1 \&$ isAllowed $=\mathrm{y}$

Astete, I. (2017). Obtenido de Epositorio UNASAAC: http://repositorio.unsaac.edu.pe/bitstream/handle/UNSAAC/2815/253T2017118 3.pdf? sequence $=1 \&$ isAllowed $=y$

Baes de datos de pueblos indigenas u originarios. (7 de 12 de 2020). Obtenido de Base de datos de pueblos indígenas u originarios: https://bdpi.cultura.gob.pe/glosario BBC. (4 de Julio de 2020). Obtenido de BBC.COM: https://www.bbc.com/mundo/noticias-52915114

Bonilla, L. (2018). Obtenido de Sentido y prácticade saberes ancestrales: https://ridum.umanizales.edu.co/xmlui/bitstream/handle/20.500.12746/3362/Tesi s_Liliana_Maria_Bonilla.pdf?sequence $=3 \&$ isAllowed $=y$

CEPAL. (2020). Obtenido de CEPAL: https://www.cepal.org/sites/default/files/news/files/folleto_amazonia_posible_y _sostenible.pdf

ContentEngine LLC, a Florida limited liability company. (2019). Obtenido de Proquest: https://www.proquest.com/wire-feeds/los-taitas-y-mamas-salasakas-compartensaberes/docview/2312467027/se-2?accountid=37408

ContentEngine LLC, a Florida limited liability company. (2020). Obtenido de Proquest: https://www.proquest.com/wire-feeds/cómo-ha-afectado-la-actual-pandemia-decovid-19/docview/2437766148/se-2 ?accountid=37408

Coronel, R. (3 de Setiembre de 2020). Obtenido de Dase UNACH: http://dspace.unach.edu.ec/bitstream/51000/7065/1/UNACH-EC-FCEHT-TGP.EDUC-2020-000054.pdf

Corporación Universitaria Minuto de Dios - UNIMINUTO. (2017). Obtenido de Proquest: https://www.proquest.com/scholarly-journals/relaciones-y-tensionesentre-la-etnoeducación-los/docview/2114614938/se-2?accountid=37408 
Delgado, M. (14 de Setiembre de 2019). Obtenido de Bibliotecas UNSA: http://bibliotecas.unsa.edu.pe/bitstream/handle/UNSA/9590/UPdelamdc.pdf?seq uence $=3 \&$ is Allowed $=\mathrm{y}$

Dueñas, Y. A. (Julio de 2017). Obtenido de TED: http://www.scielo.org.co/pdf/ted/n42/0121-3814-ted-42-00025.pdf

DW. (5 de Mayo de 2018). Obtenido de DW: https://www.dw.com/es/conocimientoancestral-ind\%C3\%ADgena-contra-el-cambio-clim\%C3\%A1tico-desdeam\%C3\%A9rica-latina/a-43673214

Flores, P. (2017). Obtenido de Saberes ancesntrales y uso de la lengua shikuana en la construccion econceptos matematicos: https://repository.upb.edu.co/bitstream/handle/20.500.11912/3337/SABERES\% 20ANCESTRALES\%20SIKUANI_\%20CONST\%20DE\%20CONCEPTOS\%20 MAT.pdf?sequence $=1 \&$ isAllowed $=\mathrm{y}$

Fonseca, K. (2017). Obtenido de Repositorio Universsidad Cesar Vallejo: http://repositorio.ucv.edu.pe/bitstream/handle/20.500.12692/23414/fonseca_ck.p df? sequence $=1 \&$ is Allowed $=\mathrm{y}$

Global Network Content Services LLC, DBA Noticias Financieras LLC. (2015). Obtenido de Proquest: https://www.proquest.com/newspapers/el-idioma-kichway-los-saberes-ancestrales-en/docview/1657811230/se-2?accountid=37408

Global Network Content Services LLC, DBA Noticias Financieras LLC. (2017). Obtenido de Proquest: https://www.proquest.com/newspapers/en-el-agro-seaplican-los-saberes-ancestrales/docview/1854601035/se-2?accountid=37408

López, L. (2017). Obtenido de Repositorio Javeriana: https://repository.javeriana.edu.co/bitstream/handle/10554/35417/Trabajo\%20de $\% 20 \mathrm{Grado} \% 20 \mathrm{No} .22$.pdf?sequence $=5 \&$ isAllowed $=\mathrm{y}$

Martinez, O., \& López, X. (27 de Setiembre de 2018). Obtenido de CIENCIA ERGO SUM: https://www.redalyc.org/jatsRepo/104/10458194012/10458194012.pdf

Melendez, E., \& Mendoza, L. (2018). Influencia de la técnica alfarera ancestral en la demanda de 1 acerámica del Pueblo de Huancas, 2017. Obtenido de http://repositorio.untrm.edu.pe/bitstream/handle/UNTRM/1655/Mendoza\%20Cr uz\%20Luis\%20$\%$ 20Melendez $\% 20$ Lopez $\% 20$ Edgar.pdf?sequence=1\&isAllowed=y 
Ministerio de Cultura. (7 de Diciembre de 2020). Obtenido de Ministerio de Cultura: https://www.gob.pe/institucion/cultura/noticias/51836-cultura-reconocimientode-diversidad-linguistica-contribuira-con-el-desarrollo-nacional

Niño, J. (2018). El legado del última Kraanti. Colombia: Revista Colombiana de Antroplogía.

Nugkuag, E. (2017). Obtenido de Cybertesis UNMSM: http://cybertesis.unmsm.edu.pe/bitstream/handle/20.500.12672/7010/Nugkuag_c e.pdf? sequence $=2 \&$ is Allowed $=y$

OIT. (2019). Obtenido de Organnización Internacina del Trabajo: https://www.ilo.org/wcmsp5/groups/public/---dgreports/---dcomm/--publ/documents/publication/wcms_713013.pdf

Osorio, A. (Junio de 2016). Obtenido de Revista Latinoamericana de Estudios Educativos: https://www.redalyc.org/pdf/1341/134149742003.pdf

Pavón, $\quad$ J. (2018). Obtenido Calle14: https://www.redalyc.org/jatsRepo/2790/279054997014/279054997014.pdf

Pérez, P. (29 de Setiembre de 2016). Obtenido de Perseitas: https://www.redalyc.org/jatsRepo/4989/498952329006/498952329006.pdf

Pérez, P. (10 de Octubre de 2018). Obtenido de Universidad Pedagógica Nacional : https://repository.cinde.org.co/bitstream/handle/20.500.11907/2628/Fundamento s\%20Tel\%c3\%baricos\%20del\%20Saber\%20Ancestral\%20Ind\%c3\%adgena.pdf ?sequence $=3 \&$ is Allowed $=\mathrm{y}$

Ramos, L. (2018). Uso de los saberes ancestrales, para el cuidado de la salud en la Comunidad Cmapesina Acoria, Huancavelica-2018. Huancayo: Unversidad Nacional del Centro del Perú.

Ramos, L. (2019). Uso de los sabares ancestrales, para cuidado de la salud en la Comunidad Campesina de Acoria, Huancavelica-2018 . Huancayo: UNCP.

Rengifo, E., Ríos, S., Fachin, L., \& Vargas, G. (15 de Abril de 2017). Obtenido de Revista Peruana de Biologia: http://www.scielo.org.pe/pdf/rpb/v24n1/a08v24n1.pdf

República, C. d. (18 de Mayo de 2006). Obtenido de Congreso: https://leyes.congreso.gob.pe/Documentos/Leyes/28736.pdf 
Salgado, E., Sandra, R., Mlaverri, L., \& Vargas, G. (2016). Saberes ancestrales el uso de la flora y fauna en lacomunidad indigena Tikuna de Cushillo Cocha, Zona fronteriza Perú Colomba Brasil. Iquitos: Revisa Peruana de Biología.

Suárez, P. (Mayo de 2019). Obtenido de Ciencia UNEMI: https://www.redalyc.org/jatsRepo/5826/582661249012/582661249012.pdf

Suarez, P. (2019). Incorporacion de los saberes ancestrales en 1 aeducacion ordinaria. Ecuador: CIENCIA UNEMI.

UNESCO. (7 de 12 de 2020). Obtenido de UNESCO: http://www.unesco.org/new/es/natural-sciences/priority-areas/links/relatedinformation/what-is-local-and-indigenous-knowledge/

$\begin{array}{llll}\text { UNICEF. } & \text { (2004). Obtenido de EIRD: }\end{array}$ https://www.eird.org/herramientas/esp/Derechos/Igualdad_con_dignidad.pdf $\begin{array}{lll}\text { UNICEF. } & \text { (2004). Obtenido de UNICEF: }\end{array}$ https://www.eird.org/herramientas/esp/Derechos/Igualdad_con_dignidad.pdf

Uribe, M. (Julio de 2019). Obtenido de Educación y ciudad: Downloads/DialnetSaberesAncestrales YTradicionales VinculadosALaPract-7390641\%20(1).pdf

Vargas, Y. (2019). Obtenido de Universidad Técnica Estatal de Quevedo: https://repositorio.uteq.edu.ec/bitstream/43000/3917/1/T-UTEQ-0041.pdf

Vela, M. (27 de Diciembre de 2019). Obtenido de Repositrio UNAP: http://repositorio.unapiquitos.edu.pe/bitstream/handle/UNAP/6796/Martin_Tesis _Titulo_2019.pdf?sequence=1\&isAllowed=y

López, L. (2017). Obtenido de Repositorio Javeriana: https://repository.javeriana.edu.co/bitstream/handle/10554/35417/Trabajo\%20de $\% 20 \mathrm{Grado} \% 20$ No.22.pdf?sequence $=5 \&$ isAllowed $=\mathrm{y}$

Martinez, O., \& López, X. (27 de Setiembre de 2018). Obtenido de CIENCIA ERGO SUM: https://www.redalyc.org/jatsRepo/104/10458194012/10458194012.pdf

Melendez, E., \& Mendoza, L. (2018). Influencia de la técnica alfarera ancestral en la demanda de 1 acerámica del Pueblo de Huancas, 2017. Obtenido de http://repositorio.untrm.edu.pe/bitstream/handle/UNTRM/1655/Mendoza\%20Cr uz\%20Luis\%20-

\%20Melendez\%20Lopez\%20Edgar.pdf?sequence=1\&isAllowed=y 
Ministerio de Cultura. (7 de Diciembre de 2020). Obtenido de Ministerio de Cultura: https://www.gob.pe/institucion/cultura/noticias/51836-cultura-reconocimientode-diversidad-linguistica-contribuira-con-el-desarrollo-nacional

Matilde López Meneses; Luisa Fernanda Gutiérrez Fuenmayor 2017 Relaciones y tensiones entre la etnoeducación y los saberes y las prácticas de los indígenas curripacos

Niño, J. (2018). El legado del última Kraanti. Colombia: Revista Colombiana de Antroplogía.

Nugkuag, E. (2017). Obtenido de Cybertesis UNMSM: http://cybertesis.unmsm.edu.pe/bitstream/handle/20.500.12672/7010/Nugkuag_c e.pdf? sequence $=2 \&$ isAllowed $=y$

OIT. (2019). Obtenido de Organnización Internacina del Trabajo: https://www.ilo.org/wcmsp5/groups/public/---dgreports/---dcomm/--publ/documents/publication/wcms_713013.pdf

Osorio, A. (Junio de 2016). Obtenido de Revista Latinoamericana de Estudios Educativos: https://www.redalyc.org/pdf/1341/134149742003.pdf

Pavón, J. (2018). Obtenido de Calle14: https://www.redalyc.org/jatsRepo/2790/279054997014/279054997014.pdf

Pérez, P. (29 de Setiembre de 2016). Obtenido de Perseitas: https://www.redalyc.org/jatsRepo/4989/498952329006/498952329006.pdf

Pérez, P. (10 de Octubre de 2018). Obtenido de Universidad Pedagógica Nacional : https://repository.cinde.org.co/bitstream/handle/20.500.11907/2628/Fundamento s\%20Tel\%c3\%baricos\%20del\%20Saber\%20Ancestral\%20Ind\%c3\%adgena.pdf ?sequence $=3 \&$ is Allowed $=\mathrm{y}$

Quiroga, Ricardo 2020. Cómo ha afectado la actual pandemia de Covid-19 los ritos y saberes ancestrales.

(2018). Obtenido de Dispace.unitru: http://www.dspace.unitru.edu.pe/bitstream/handle/UNITRU/11888/Mera\%20An drade \%20Rafael\%20Isa\%c3\%adas.pdf?sequence=1\&isAllowed=y

Abarca, R. (2017). Obtenido de Repositorio Universidad Nacinal San Anatonio Abad del 
http://repositorio.unsaac.edu.pe/bitstream/handle/UNSAAC/2815/253T2017118 3.pdf?sequence $=1 \&$ is Allowed $=\mathrm{y}$

Astete, I. (2017). Obtenido de Epositorio UNASAAC: http://repositorio.unsaac.edu.pe/bitstream/handle/UNSAAC/2815/253T2017118 3.pdf? sequence $=1 \&$ isAllowed $=\mathrm{y}$

Baes de datos de pueblos indigenas u originarios. (7 de 12 de 2020). Obtenido de Base de datos de pueblos indígenas u originarios: https://bdpi.cultura.gob.pe/glosario

BBC. (4 de Julio de 2020). Obtenido de BBC.COM: https://www.bbc.com/mundo/noticias-52915114

Bonilla, L. (2018). Obtenido de Sentido y prácticade saberes ancestrales: https://ridum.umanizales.edu.co/xmlui/bitstream/handle/20.500.12746/3362/Tesi s_Liliana_Maria_Bonilla.pdf?sequence=3\&isAllowed=y

CEPAL. (2020). Obtenido de CEPAL: https://www.cepal.org/sites/default/files/news/files/folleto_amazonia_posible_y _sostenible.pdf

ContentEngine LLC, a Florida limited liability company. (2019). Obtenido de Proquest: https://www.proquest.com/wire-feeds/los-taitas-y-mamas-salasakas-compartensaberes/docview/2312467027/se-2?accountid=37408

ContentEngine LLC, a Florida limited liability company. (2020). Obtenido de Proquest: https://www.proquest.com/wire-feeds/cómo-ha-afectado-la-actual-pandemia-decovid-19/docview/2437766148/se-2?accountid=37408

Coronel, R. (3 de Setiembre de 2020). Obtenido de Dase UNACH: http://dspace.unach.edu.ec/bitstream/51000/7065/1/UNACH-EC-FCEHT-TGP.EDUC-2020-000054.pdf

Corporación Universitaria Minuto de Dios - UNIMINUTO. (2017). Obtenido de Proquest: https://www.proquest.com/scholarly-journals/relaciones-y-tensionesentre-la-etnoeducación-los/docview/2114614938/se-2?accountid=37408

Delgado, M. (14 de Setiembre de 2019). Obtenido de Bibliotecas UNSA: http://bibliotecas.unsa.edu.pe/bitstream/handle/UNSA/9590/UPdelamdc.pdf?seq uence $=3 \&$ is Allowed $=\mathrm{y}$

Dueñas, Y. A. (Julio de 2017). Obtenido de TED: http://www.scielo.org.co/pdf/ted/n42/0121-3814-ted-42-00025.pdf 
DW. (5 de Mayo de 2018). Obtenido de DW: https://www.dw.com/es/conocimientoancestral-ind\%C3\%ADgena-contra-el-cambio-clim\%C3\%A1tico-desdeam\%C3\%A9rica-latina/a-43673214

Flores, P. (2017). Obtenido de Saberes ancesntrales y uso de la lengua shikuana en la construccion econceptos matematicos: https://repository.upb.edu.co/bitstream/handle/20.500.11912/3337/SABERES\% 20ANCESTRALES\%20SIKUANI_\%20CONST\%20DE\%20CONCEPTOS\%20 MAT.pdf?sequence=1\&isAllowed=y

Fonseca, K. (2017). Obtenido de Repositorio Universsidad Cesar Vallejo: http://repositorio.ucv.edu.pe/bitstream/handle/20.500.12692/23414/fonseca_ck.p df? sequence $=1 \&$ is Allowed $=y$

Global Network Content Services LLC, DBA Noticias Financieras LLC. (2015). Obtenido de Proquest: https://www.proquest.com/newspapers/el-idioma-kichway-los-saberes-ancestrales-en/docview/1657811230/se-2?accountid=37408

Global Network Content Services LLC, DBA Noticias Financieras LLC. (2017). Obtenido de Proquest: https://www.proquest.com/newspapers/en-el-agro-seaplican-los-saberes-ancestrales/docview/1854601035/se-2?accountid=37408

López, L. (2017). Obtenido de Repositorio Javeriana: https://repository.javeriana.edu.co/bitstream/handle/10554/35417/Trabajo\%20de $\% 20 \mathrm{Grado} \% 20$ No.22.pdf?sequence=5\&isAllowed=y

Martinez, O., \& López, X. (27 de Setiembre de 2018). Obtenido de CIENCIA ERGO SUM: https://www.redalyc.org/jatsRepo/104/10458194012/10458194012.pdf

Melendez, E., \& Mendoza, L. (2018). Influencia de la técnica alfarera ancestral en la demanda de 1 acerámica del Pueblo de Huancas, 2017. Obtenido de http://repositorio.untrm.edu.pe/bitstream/handle/UNTRM/1655/Mendoza\%20Cr uz\%20Luis\%20$\%$ 20Melendez\%20Lopez\%20Edgar.pdf?sequence=1\&isAllowed=y

Ministerio de Cultura. (7 de Diciembre de 2020). Obtenido de Ministerio de Cultura: https://www.gob.pe/institucion/cultura/noticias/51836-cultura-reconocimientode-diversidad-linguistica-contribuira-con-el-desarrollo-nacional

Niño, J. (2018). El legado del última Kraanti. Colombia: Revista Colombiana de Antroplogía. 
Nugkuag, E. (2017). Obtenido de Cybertesis UNMSM: http://cybertesis.unmsm.edu.pe/bitstream/handle/20.500.12672/7010/Nugkuag_c e.pdf?sequence $=2 \&$ isAllowed $=y$

OIT. (2019). Obtenido de Organnización Internacina del Trabajo: https://www.ilo.org/wcmsp5/groups/public/---dgreports/---dcomm/--publ/documents/publication/wcms_713013.pdf

Osorio, A. (Junio de 2016). Obtenido de Revista Latinoamericana de Estudios Educativos: https://www.redalyc.org/pdf/1341/134149742003.pdf

Pavón, J. (2018). Obtenido de Calle14: https://www.redalyc.org/jatsRepo/2790/279054997014/279054997014.pdf

Pérez, P. (29 de Setiembre de 2016). Obtenido de Perseitas: https://www.redalyc.org/jatsRepo/4989/498952329006/498952329006.pdf

Pérez, P. (10 de Octubre de 2018). Obtenido de Universidad Pedagógica Nacional : https://repository.cinde.org.co/bitstream/handle/20.500.11907/2628/Fundamento s\%20Tel\%c3\%baricos\%20de1\%20Saber\%20Ancestral\%20Ind\%c3\%adgena.pdf ?sequence $=3 \&$ is Allowed $=\mathrm{y}$

Ramos, L. (2018). Uso de los saberes ancestrales, para el cuidado de la salud en la Comunidad Cmapesina Acoria, Huancavelica-2018. Huancayo: Unversidad Nacional del Centro del Perú.

Ramos, L. (2019). Uso de los sabares ancestrales, para cuidado de la salud en la Comunidad Campesina de Acoria, Huancavelica-2018 . Huancayo: UNCP.

Rengifo, E., Ríos, S., Fachin, L., \& Vargas, G. (15 de Abril de 2017). Obtenido de Revista Peruana de Biologia: http://www.scielo.org.pe/pdf/rpb/v24n1/a08v24n1.pdf

República, C. d. (18 de Mayo de 2006). Obtenido de Congreso: https://leyes.congreso.gob.pe/Documentos/Leyes/28736.pdf

Salgado, E., Sandra, R., Mlaverri, L., \& Vargas, G. (2016). Saberes ancestrales el uso de la flora y fauna en lacomunidad indigena Tikuna de Cushillo Cocha, Zona fronteriza Perú Colomba Brasil. Iquitos: Revisa Peruana de Biología.

Suárez, P. (Mayo de 2019). Obtenido de Ciencia UNEMI: https://www.redalyc.org/jatsRepo/5826/582661249012/582661249012.pdf 
Suarez, P. (2019). Incorporacion de los saberes ancestrales en 1 aeducacion ordinaria. Ecuador: CIENCIA UNEMI.

UNESCO. (7 de 12 de 2020). Obtenido de UNESCO: http://www.unesco.org/new/es/natural-sciences/priority-areas/links/relatedinformation/what-is-local-and-indigenous-knowledge/

UNICEF. (2004). Obtenido de EIRD: https://www.eird.org/herramientas/esp/Derechos/Igualdad_con_dignidad.pdf UNICEF. (2004). Obtenido de UNICEF: https://www.eird.org/herramientas/esp/Derechos/Igualdad_con_dignidad.pdf

Uribe, M. (Julio de 2019). Obtenido de Educación y ciudad: Downloads/DialnetSaberesAncestrales YTradicionalesVinculadosALaPract-7390641\%20(1).pdf

Vargas, Y. (2019). Obtenido de Universidad Técnica Estatal de Quevedo: https://repositorio.uteq.edu.ec/bitstream/43000/3917/1/T-UTEQ-0041.pdf

Vela, M. (27 de Diciembre de 2019). Obtenido de Repositrio UNAP: http://repositorio.unapiquitos.edu.pe/bitstream/handle/UNAP/6796/Martin_Tesis _Titulo_2019.pdf?sequence=1\&isAllowed=y 